Band so stark in Anspruch genommen wie Maurice Duverger. Als hätte eine ganze Forschungsrichtung nichts anderes zu tun, als immer wieder die Thesen zu widerlegen, von denen sich Duverger vielfach selbst distanziert hat. Erfreulich ist natürlich, dass etliche frühere Forschungsergebnisse bestätigt werden, unter anderem die These des Rezensenten aus dem Jahre 1984, dass grundlegende Wahlsystemwechsel rar sind. Man sollte sich jedoch davor hüten, den national begrenzten Vergleich, wie Matthew S. Shugart (S. 29), auch als komparativ zu bezeichnen, der im Grunde deshalb vorzuziehen sei, weil in ihm - anders als im Ländervergleich - viele Variablen konstant gehalten werden könnten. Zum einen wird vernachlässigt, worin Kunst und Gewinn komparativer Forschung liegen: die Kunst im qualitativen Forschungsdesign ausgesuchter Fälle, die ein analytisch fruchtbares Maß an Ähnlichkeiten und Unterschieden aufweisen, der Gewinn in der näheren Bestimmung jener gesellschaftlichen und politischen Bedingungen, unter denen Wahlsysteme diese oder jene Auswirkungen zeigen. In einer qualitativ vergleichenden Analyse werden dabei unabdingbar die wechselhaften Konsequenzen der Kontextfaktoren thematisiert, die in einer nationalen Untersuchung leichthin als konstant angenommen werden und dann systematisch außerhalb der Untersuchung bleiben. Zum anderen ist es diese Annahme selbst, dass der nationale Kontext für die Wahlsystemanalyse über längere Zeit konstant bleibe, die zu den üblichen Reduktionismen der Wahlsystemforschung gehört.

Dieter Nohlen

\title{
Prominente Studie zu politischen Milieus
}

Neugebauer, Gero: Politische Milieus in Deutschland. Die Studie der Friedrich-Ebert-Stiftung, Verlag J.H.W. Dietz Nachfolger, Bonn 2007, 145 Seiten, € 9,90.

Sozialwissenschaftlichen Studien wird in der Regel nur begrenzte Aufmerksamkeit geschenkt. Einige Arbeiten erfahren eine gewisse fachinterne Resonanz, aber nur ganz wenige werden von einer breiteren Öffentlichkeit wahrgenommen oder gar Gegenstand politischer Diskussionen. Dass aber eine Untersuchung bereits vor ihrem eigentlichen Erscheinen eine unmittelbare Reaktion des Vizekanzlers hervorruft, ist wohl ein Unikum und besonderen Entstehungszusammenhängen geschuldet. Im Herbst 2006 fügte sich der Befund einer von der Friedrich-Ebert-Stiftung in Auftrag gegebenen Studie, wonach acht Prozent der Bundesbürger dem „abgehängten Prekariat" ${ }^{\text {" }}$ angehören, genau in die vom damaligen SPDVorsitzenden Kurt Beck initiierte so genannte Unterschichten-Diskussion. Die Untersuchung wurde als Beleg für die Verschärfung sozialer Probleme infolge der von der SPD zu verantwortenden „Agenda 2010“-Politik gewertet. Der damalige Arbeits- und Sozialminister Franz Müntefering sah sich daraufhin zu der Aussage veranlasst, es gebe keine Schichten in Deutschland, sondern lediglich Menschen, die schwächer seien und es schwerer hätten.

Nachdem die öffentliche Aufmerksamkeit sich wieder anderen Themen zugewendet hat, lohnt es sich, jenseits tagespolitischer Aufgeregtheiten einen zweiten Blick auf die Studie

1 So die Begriffsbildung von Rita Müller-Hilmer vom Institut TNS Infratest Sozialforschung, das wesentlich an der Studie beteiligt war. 
der Friedrich-Ebert-Stiftung zu werfen. Deren Ziel war es, das Verhältnis der Bundesbürger zu sozialen und wirtschaftlichen Reformen auszuloten und auf sozialwissenschaftlich fundierter Basis Empfehlungen für die politische Praxis abzuleiten. Dabei wurde der Versuch unternommen, eine aktuelle politische „Anatomie des deutschen Elektorats“ (S. 8) vorzulegen. Als Basis dienten zwei qualitative Studien des Institutes für Zielgruppenkommunikation und des Sinus-Instituts, die sich auf Einzelexplorationen und Gruppendiskussionen stützten. Anschließend führte TNS Infratest Sozialforschung eine für die wahlberechtigte Bevölkerung repräsentative Face-to-face-Befragung durch (S. 22 bis 26). Gero Neugebauer kommt das Verdienst zu, innerhalb kürzester Zeit die Einzelstudien zusammengeführt und für eine Publikation aufbereitet zu haben.

In seiner Darstellung klärt Neugebauer einleitend einige grundlegende soziologische Begriffe (Modernisierung, sozialer Wandel, Wertewandel etc., Kap. 2). Wesentlich für die weitere Analyse ist insbesondere das Konzept sozialer Milieus, demzufolge gegenwärtige Gesellschaften sowohl vertikal als auch horizontal ausdifferenziert sind.

Die Präsentation der Befragungsergebnisse befasst sich zunächst mit der gesellschaftlichen Grundstimmung (Kap. 3). Die Umfragen belegen, dass gegenwärtig ein Gefühl der Verunsicherung in verschiedenen Bereichen vorherrscht. Ursache hierfür ist die sich - laut Selbstauskunft - verschlechternde finanzielle Situation vieler Bürger (S. 29). Zugleich werden zunehmende soziale Unterschiede konstatiert (S. 28 f.) und die Möglichkeiten zum gesellschaftlichen Aufstieg als eher gering eingeschätzt (S. 46). Damit korrespondiert der Befund, wonach eine deutliche Mehrheit der Befragten soziale Werte - im Vergleich zu marktliberalen - präferiert (S. 49 und S. 59) und hohe Erwartungshaltungen an den Staat richtet. Da diese Gerechtigkeits- und Sicherheitsbedürfnisse (S. 61) aber als nicht eingelöst gelten, fällt die Haltung vieler Bürger zur Politik recht kritisch aus (S. 30). Im Anschluss an die Diskussionen zum schwindenden Sozialkaptial und zum Rückgang bürgerschaftlichen Engagements kontrastiert Neugebauer die Unzufriedenheit über die politischen Gegebenheiten mit der wachsenden Orientierung auf das private Umfeld aus Familie und Freunden (S. 39).

Im nächsten Schritt unternimmt die Studie den Versuch, eine Topographie politischsozialer Strukturen in Deutschland zu zeichnen. Demnach lassen sich - ermittelt durch die Position auf drei Wertekonfliktebenen - neun verschiedene Milieus unterscheiden. Hierunter fällt auch das zu einiger Prominenz gelangte „abgehängte Prekariat“, dem acht Prozent, in den neuen Ländern gar zwanzig Prozent der deutschen Bevölkerung zugerechnet werden. Die Konstruktion und die Einteilung der Milieus können nicht durchgehend überzeugen. Zum einen ist nur in Teilen ausgewiesen, aufgrund welcher Methoden und Berechnungen die verschiedenen Milieus zustande gekommen sind. Zum anderen fehlt weitgehend der Anschluss an diverse Vorläuferstudien (etwa die einschlägigen Untersuchungen von Michael Vester). Für Parteienforscher von einiger Relevanz ist jedoch, dass gerade Angehörige des „abgehängten Prekariats“ einen erheblichen Teil der Wählerschaft rechter und linker Parteien stellen und das untere gesellschaftliche Drittel überproportional zur Nichtwahl tendiert (S. 100). Ferner zeigt sich, dass die Anhängerschaften von FDP und Grünen zu großen Teilen aus spezifischen Milieus des oberen Drittels bestehen, während Union und insbesondere SPD über die diversen Milieus hinweg Wähler mobilisieren. Zudem bestätigt die Studie den bekannten Befund, dass die Zugehörigkeit zu und die Partizipationsbereitschaft in Parteien mit dem sozialen Status steigt (S. 98). In der Gesamtschau zeichnet die Untersuchung folglich das Bild einer stark segmentierten Gesellschaft, wobei es in allen 
Milieus (vor allem aber in den sozial schwächeren) Unzufriedenheit, Zukunftsängste und Politikverdrossenheit gibt (S. 106).

Dies beeinflusst auch die im nächsten Schritt erfasste Reformhaltung vieler Bürger. Demnach ist zwar eine erstaunlich hohe und mit dem sozialen Status steigende Veränderungsbereitschaft zu verzeichnen (S. 107). Die Erwartungen hinsichtlich der tatsächlich durchgeführten Reformen sind jedoch gering - insbesondere mit Blick auf sozialstaatliche Maßnahmen. Entsprechend pessimistisch schätzt auch der Großteil der Befragten den Nutzen der bisherigen Reformbemühungen ein (S. 111). Deren Notwendigkeit wird mithin von den Bürgern eingesehen, den bisherigen Ergebnissen und künftigen Reformen stehen sie allerdings eher skeptisch gegenüber. Dahinter steht ein grundlegender Wandel: Wurden früher unter Reformen vornehmlich wohlfahrtsstaatliche Umverteilungsmaßnahmen nach unten verstanden, so gelten sie heutzutage vielen als Synonym für die Reduzierung staatlicher Transfers und die Stärkung eigenverantwortlicher Anteile (S. 130).

Aus diesen Ergebnissen und auf der Basis der qualitativen Untersuchungsabschnitte entwickelt Neugebauer Empfehlungen für die politische Kommunikation. Als dringlich erscheint ihm, dass die Reformdebatten immer eine Erfolgsperspektive aufweisen und bei den Problemen der Bürger ansetzen (S. 128). Die bisherigen Kommunikationsbemühungen wertet Neugebauer als defizitär. Mit anderen konstatiert er eine mangelhafte Repräsentation gesellschaftlicher Interessen durch die Parteien (S. 138).

Aufgrund ihres Entstehungszusammenhangs ist die Publikation sicherlich nicht an den gleichen Maßstäben zu messen wie herkömmliche sozialwissenschaftliche Studien. Aber ihre Wirkungsgeschichte und einige ihrer Befunde sollten unbedingt wahrgenommen werden, da sie wesentliche Probleme in den Beziehungen zwischen Bürgern, Parteien und Politik sowie Schwachstellen in der Vermittlung sozialwissenschaftlicher Erkenntnisse in die breitere Öffentlichkeit aufzeigen: Die Arbeit akzentuiert die Unzufriedenheit vieler Bürger mit den gegenwärtigen sozialen, wirtschaftlichen und politischen Gegebenheiten, die ungeachtet unbestreitbarer Erfolge - etwa auf dem Arbeitsmarkt - besteht. Der Vergleich zu vergangenen Befragungswerten und Befunden, die für viele der behandelten Bereiche zur Verfügung stehen, hätte die Einordnung und Bewertung der aktuellen Umfrageergebnisse nochmals erleichtert. Nichtsdestoweniger ist die Politik und sind insbesondere die Parteien angehalten, unter Berücksichtigung einiger der empfohlenen Maßnahmen zur besseren Kommunikation sozialer und wirtschaftlicher Reformen und Reformerfolge beizutragen. Vor allem wird es darauf ankommen, auf die Ziel- und Sinnerwartungen vieler Bürger authentisch und glaubwürdig zu reagieren.

Die große Beachtung, die den ersten Ergebnissen der Studie geschenkt wurde, verdeutlicht das Aufmerksamkeitspotential, das Befunden und Thesen der Sozialwissenschaften zukommen kann. Es ist deren Aufgabe, dem Orientierungs- und Deutungsbedürfnis einer größeren Öffentlichkeit Rechnung zu tragen und sich an den Debatten um den gegenwärtigen Zustand der Gesellschaft mit wissenschaftlich begründeten Interpretationen zu beteiligen. Die vorliegende Studie regt dazu an, diese Aufgabe ernster zu nehmen und engagierter anzugehen, als dies in der Vergangenheit häufig der Fall war. Das entsprechende Wissen und die einschlägigen Einsichten stehen in den Sozialwissenschaften jedenfalls zur Verfügung - sie sollten zukünftig auch stärker genutzt werden. 